\title{
Respuesta inmune celular como indicador biológico de deficiencia de zinc: en pacientes con fenilquetonuria
}

\author{
Marianela Arévalo N. ${ }^{\text {; }}$ Valeska Simón Z. ${ }^{2}$; Marcia Cortés G. ${ }^{1 *}$ \\ Verónica Cornejo E. ${ }^{3}$ Liana Schlesinger $F^{4}$

\section{Cell-mediated inmune response as a biological indicator of zinc deficiency: phenylketonuria as a study model}

\begin{abstract}
Contradictory information exists in relation to zine nutritional status in phenylketonuria. The objetive of this study was to confirm thot there it is indirect evidence of zinc deficiency in polienls with phenylkelonurio as meosured by the effect of zinc supplementation on cellmedialed inmune response. Delayed cutoneous hypersensilivity reodion and lymphoproliferative response to phytohemagglutinin was delermined in eight phenylketonuric children under specific nutritional management (low phenylalanine diet) before and after zinc acelote supplementolion. Two of eight patients had a positive cutaneous response at odmission and six became posiltve alter zinc supplemenlation. This difference was stalislicolly significont ip $<0.05 \mathrm{Mac}$ Nemor lest\}. Lymphoprolilerolive iesponse 10 PHA was olso significantly increased at the end of zinc

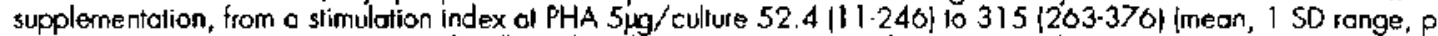
$<0.05$ Tparl. The improvemenl of cell mediated inmune response afier zinc supplementalion, suggests thal zinc deficiency is present in phenylketonutic pallents treated with low phenylalanine diets.
\end{abstract}

(Key wordst cell-mediated inmune response, zinc nutritionol slotus, phenylketonurio, phenylalanine.)

La fenilquetonuria (PKU) es una enfermedad metabólica que se caracteriza por ausencia de actividad de la enzima fenilalaninahidroxilasa, que determina una incapacidad de transformar fenilalanina en tirosina. Cuando el diagnóstico y tratamiento de estos pacientes no se realiza precozmente se produce daño cerebral severol, 2 . Se ha demostrado que dietas pobres en fenilalanina son el tratamiento adecuado para los sujetos con $\mathrm{PKU}^{3}$. El contenido de proteínas y minerales traza en este tipo de dietas no salisfacen los requerimientos nutricionales de estos pacientes, lo que

1. Tecnólogo Médico. Unidad de Inmunologia, Instituto de Nutrición y Tecnología de los Alimentos, Universidad de Chile.

2. Técrico Químico Laboratorisla. Unidad de Inmunología, Instituto de Nutrición y Tecnología de los Alimentos, Universidad de Chile.

3. Nutricionisı. Unidad de Genética y Enfermedades Metabólicss. Instjuto de Nutrición y Tecnología de los A limentos, Universidad de Chile.

4. Médico, Unidad de Inmunología, Insututo de Nutrición y Tecrología de los Alimentos, Universidad de Chile. se traduce en una desnutrición secundaria, que se acompafia de deficiencia de minerales como zinc y hiemo, entre otros ${ }^{4}$. En la fenilquetonuria existen datos contradictorios en cuanto a nutrición de zinc. Algunos estudios demuestran niveles de zinc plasmático disminuidos en estos pacientes ${ }^{5}$. en cambio otros muestran niveles normales de este mineral traza ${ }^{6}$. Los criterios usados en la actualidad para hacer el diagnóstico de deficiencia de zinc han sido básicamente las mediciones de zinc en pelo y plasma y la respuesta biologica frente a la administración de este mineral traza. El zinc desempeña un rol fundamental sobre la respuesta inmune, su deficiencia determina atrofia del tejido linfoide, negativización de las reacciones cutáneas de hipersensibilidad retardada, disminución de la capacidad proliferativa de linfocitos frente a mitógenos y antígenos, alteración de la quimiotaxis de polimorfonucleares y monocitos ${ }^{7}$. Los efectos de la deficiencia de zinc han sido estudiados especialmente en acrodermatitis enteropática, enfermedad congénita en que existe un defecto de la absorción de zinc. Esta afección se acompaña de inmunodeficiencia, la 
que se corrige con la administración de este mineral traza.9. También se ha observado deficiencia de zinc en pacientes que reciben hiperalimentación parenteral prolongada, en el paciente anciano y en sujetos con inmunodeficiencia común variable ${ }^{10}, 11$.

El objetivo del presente estudio fue confirmar si existe evidencia indirecta de délicit de zinc en pacientes con PKU, evaluando el efecto gue ejerce la suplementación de zinc sobre la respuesta inmune celular.

\section{Material y Método}

Se evaluó la respuesta inmune celular en ocho niños fenitquetonúricos antes y después de tres meses de suplementación con acetato de zinc, en dosis de $0,5 \mathrm{mg} \mathbf{~ k g}$-dia administrado entre las comidas. El grupo en estudio estaba constituido por ocho pacientes fenilquetonúricos, cinco hombres, cuyas edades fluctuaban entre 2 y 18 affos. Todos los pacientes ejan controlados en la policlínica de enfermedades metabólicas del Instituto de Nutrición y Tecnología de los Alimentos de la Universidad de Chile (INTA), donde se les indicó una dieta pobre en fenilalanina. Esta consistía en leche de vaca en polvo diluida (Purita) que proporcionaba 0,7 a I g de proleína-kg-día y un aporte calórico de 90 a 140 calorías kg día, para lo cual los niños recibieron suplementos de maltosa dextrina hasta $20 \%$ del total de calorías. Todos los pacientes recibieron permanentemente suplemenlos de hierro, calcio y vítaminas. Se evaluó el estado nutri cional deteminando peso y talla (mediciones efectuadas por una enfermera universitaria) al ingreso y después de tres meses de suplementación con zinc. Los resultados se analizaron empleando el porcentaje de adecuación peso para edad (P/E) y talla para edad (T/E) utilizando las tablas del NCIIS. Se midieron los niveles de fenitalanina por el método de Guthrie ${ }^{12}$ antes y después de finalizada la suplementación con zinc.

La respuesta inmune celular se evaluó medjante prueba cutárea de sensibilidad retardada, respuesta linfoproliferativa a fitohemaglutinina y deterninsción de subpoblacjones linfocitarias. La prueba de hipersensibilidad retatdada se reslizó mediante el Multitest CMI de Rhodia Meriux ${ }^{13}$, que contiene un conjunto de 7 antígenos (proteus, tricofitón. candida, tćtanos, difteria, estreptococo y tuberculina) y un control de glicerina; se consideró como respuesta posiliva la presencia una induración $>2 \mathrm{~mm}$ a tres o más de los antígenos empleados, $48 \mathrm{~h}$ después de su aplicación. La capacidad de respuesta proliferaliva de los linfocitos frente a filohemaglutinina ( $\mathrm{PH} A$ ) se realizó previa separaciốn de lcucocitos por gradiente de Ficoll Hypaque. Los linfocitos se cultivaron en presencia de diferentes concentraciones de PIIA a $37^{\circ} \mathrm{C}$ en ambiente húmedo, $95 \%$ de aire y $5 \%$ de $\mathrm{CO}_{2}$. Como control se realjzaron cullivos sin estimular en las mistnas condiciones antes señaladas y todos los cultivos se ectuaron en triplicado. La capacidad proliferativa se determino midiendo la incorporación de timidina tritiada a DNA celular y los resultados se expresaton como índice de estimulación, que es la razón entre cuentas por minuto (cpm) en cullivos estimulados con PHA y cpm en cultivos sin estimular ${ }^{14}$. Las subpoblaciones de linfocitos T se cuandifi caron incubando 0,5-106 linfocitos en medio RPMI 1640 suplementado con $1 \%$ de albúmina, en presencia de anticuerpos monoclonales DAKO-T3 para linfocitos $\mathrm{T}$ totales, DAKO-T4 para linfocitos ayudadores y DAKO-T8 para linfocitos $T$ supresores/citotóxicos (Dako Corporation) por un período de $60 \mathrm{~min}$ a $4^{\circ} \mathrm{C}$; después de tres lavados cl complejo se incubó con un anticuerpo conjugado con fluoresceina (Kallestad) durante $40 \mathrm{~min}$ a $4^{\circ} \mathrm{C}$ y se deteminó el número de linfocitos marcados con fluoresceína contando un total de 200 células ${ }^{15} .16$.

El análisis estadístico de los datos se realizo con el paquete estadístico SAS (Institute Inc, Cary, NC). Se emplearon pruebas paradas de I y ensayo de Mac Nemar.

El estudio fue aprobado por el Comité de Etica del Instituto de Nutrición y Tecnología de los Alimentos y la incorporación de los pacientes al mismo se realizó con consentimiento informado de los padres.

\section{Resultados}

Las relaciones $\mathrm{P} / \mathrm{E}$ y $\mathrm{T} / \mathrm{E}$ y la fenilalanina sérica se mantuvieron constantes entre el inicio y el término de la suplementación con zinc (tabla 1 ).

Antes de la suplementación de la dieta con zinc, solo dos pacientes mostraban respuestas positivas de hipersensibilidad cutánea retardada, número que aumentó a ocho después de recibir zinc $(p<0,05)$.

Se observó un claro aumento de la respuesta linfoproliferativa a PHA al término de la suplementación con zinc (tabla 2), estadísticamente significativo en las concentraciones de 2,$5 ; 5$ y $10 \mu \mathrm{g}$ de PHA por cultivo. El número de linfocitos $\mathrm{T}$ totales, $\mathrm{T}$ ayudadores y $\mathrm{T}$ supresores/citotóxicos no presentó variaciones durante el estudio, mantenićndose sus valores dentro del margen normal para la edad en todos los pacientes evaluados.

\section{Comentario}

Este estudio sugicre que los pacientes con PKU presentan déficit de zinc, por el aumento de la respuesta linfoproliferativa frente a PHA y la positivización de las reaccioncs cutáneas de hipersensibilidad retardada, después de suministrar zinc.

Existe escasa información sobre la respucsta inmune celular en pacientes con PKU. El único trabajo publicado muestra que no existe altera- 
Tabla 1

Características clínicas y de laboratorio de los pacientes fenilq̣uetonúricos

\begin{tabular}{|c|c|c|c|c|c|c|c|c|}
\hline \multirow[b]{2}{*}{ Paciente } & \multirow[b]{2}{*}{ Sexo } & \multirow[b]{2}{*}{$\begin{array}{c}\text { Edzd } \\
\text { (años) }\end{array}$} & \multicolumn{3}{|c|}{ Antes de zlnc } & \multicolumn{3}{|c|}{ Después de zinc } \\
\hline & & & $\begin{array}{l}\text { Fenifalanina* } \\
\left.\text { (mg } x_{0}\right)\end{array}$ & P/E & $\mathrm{T} / \mathrm{E}^{* *}$ & $\begin{array}{l}\text { Fenilalanina } \\
\text { (mg } \%)\end{array}$ & $\mathrm{P} / \mathrm{E}$ & $\mathbf{T} / \mathbf{E}$ \\
\hline 1 & M & 3 & 28 & 91,9 & 99,3 & $18 \cdot 20$ & 91,8 & 96.2 \\
\hline 2 & F & 6 & $>20$ & 98,0 & 94,3 & $14-16$ & 98.1 & 93,7 \\
\hline 3 & $\mathbf{M}$ & 14 & $>20$ & 71,3 & 90,1 & $>20$ & 70,1 & 89.5 \\
\hline 4 & $M$ & 6 & $12-14$ & 78,7 & 92,8 & $>22$ & 74,2 & 91,6 \\
\hline 5 & $\mathbf{F}$ & 2 & $2-4$ & 70,1 & 90,0 & $2-4$ & 88,6 & 92.8 \\
\hline 6 & M & 3 & $10 \cdot 12$ & 78,1 & 90,4 & $18-20$ & 80,4 & 91,2 \\
\hline 7 & $\mathbf{M}$ & 18 & $>20$ & 61.7 & 87.0 & $>20$ & 64,8 & 87,0 \\
\hline 8 & $\mathrm{~F}$ & 13 & $8-10$ & 79,4 & 91,0 & $8-10$ & 82,7 & 90,6 \\
\hline
\end{tabular}

- Valores aceprables de fenilalanina sérica en nifhos con fenilquetonuria según edad:

$<10 \mathrm{mg} \%$ para menores de 10 años, entre 15 y $20 \mathrm{mg} \%$ de 10 a 25 años.

** PJE y T/E calculado por cl porcentaje de adecuación al percentil 50 de las tablas NCHS.

Tabla 2

Respuesta linfoproliferativa a fitohema glutininaen pacientes fenilquetonúricos

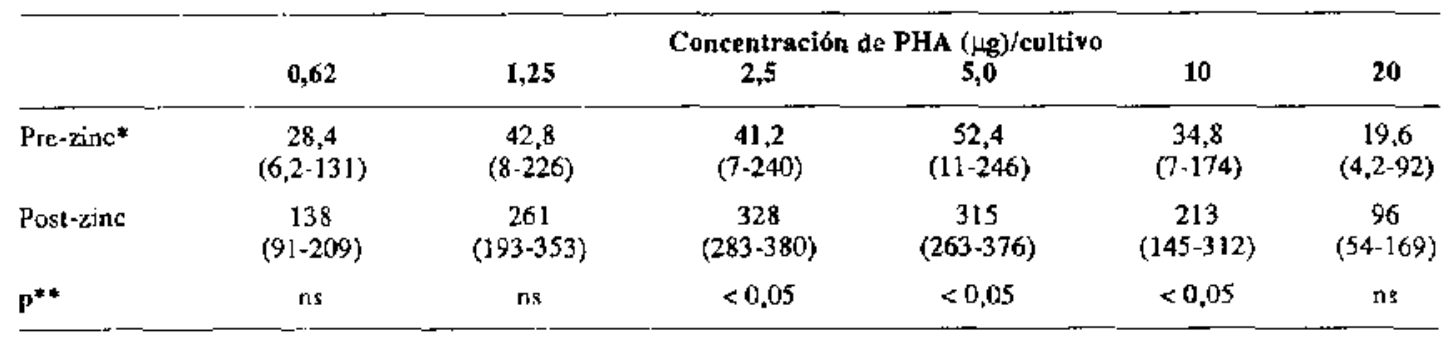

* Resultados expresados como indice de estimulación. Las cifras corresponden al promedio geométrico, entre paréntesis el margen de una desviación estándar.

* Prueba de t-pareado.

ción de la respuesta cutảnea de hipcrsensibilidad retardada, del número de linfocitos $\mathrm{T}$ y de la capacidad proliferativa de linfocitos frente a PHA $^{17}$. Sin embargo, los investigadores no realizaron una curva dosis-respuesta e incluso no señalan la concentración de mitógeno utilizado ${ }^{17}$. En nuestro estudio realizamos una curva dosis-respuesta frente a PHA en cada paciente, antes y después de la suplementación con zinc. La respuesta obtenida a continuación del suministro del mineral fue similar a la observada en 12 sujetos controles adultos cutróficos sanos, cuyo índice de estimulación, expresado como promedio geométrico y margen del 1 DE fue 149 (98-228).

Otro hecho interesante es que, después de la suplementación con zinc, en $75 \%$ de los niños se hizo positiva su respuesta cutánea de hipersensibilidad retardada. Cabe senalar que los pacientes no recibieron vacunas, ni sufrieron enfermedades intercurrentes durante el período en que se realizó el estudio. Se utilizó la prucba múltiple con siete antígenos diferentes, con el objeto de obviar el efecto reforzador que pudiera determinar el uso aislado del PPD. Sin embargo, se ha señalado que no existírían evidencias definitivas de que la repctición de una prueba cutánea provoque positivización de la reacción cutánca de hipersensibilidad retarda$\mathrm{da}^{18}$.

Estos hallazgos sugieren que los pacientes fenilquetonúricos presentan deficiencia de zinc. En nuestro estudio no fue posible medir la con- 
centración de zinc en suero, sin embargo ćsta no es un indicador que permita definir el estado nutricional de zinc, ya que infecciones leves o procesos inflamatorios determinan hipocinquemia en ausencia de deficiencia real de ese elemento. Por otra parte, cuando la deficiencia existe, aumenta el catabolismo celular, Jo que causa liberación de este elemento desde los tejidos al plasma, enmascarando la carencia. La medición de zinc en el cabello no es un método confiable para evaluar el estado nutricional de este mincral traza, puesto que factores como el crecimiento del pelo y el uso de ciertos champúes pueden afectar su medición' 19 . Un buen indicador del estado nutricional de zinc, es la respuesta biológica secundaria a su administración, método utilizado en este estudio. Nuestros resultados son concordantes con los obtenidos en un estudio de suplementación de zinc en lactantes con desnutrición calórico proteica, en que se describió que la concentración de zinc en plasma y en los leucocitos polimorfonucleares no se modifić substancialmente durante la rehabilitación nutricional con leche fortificada con zinc ${ }^{20}$. Estc mineral traza determinó importantes efectos biológicos que se expresaron como transformación posiliva de las reacciones cutáneas, aumento de la respuesta linfoproliferativa frente a PHA, aumento en la concentración de la IgA secrctora e incremento signíficativo de la talla ${ }^{20}$. La alteración de la respuesta inmune celular en PKU no sería producida por disminución en el número de los linfocitos $T$, sino por cambios en la función de estas células. La presencia de zinc es fundamental para que se produzca replicación del DNA frente a estimulación con míógenos, puesto que enzimas tales como DNA polimerasa y Iranscriptasa reversa utilizan este mineral como $\operatorname{cofactor}^{21,22}$. Quelantes del zinc inhiben la actividad de estas cnzimas y a la inversa la suplementación con este mineral traza revicrte esta inhibición ${ }^{22}$. La estimulación de la respuesta inmunitaria celular podría alribuirse a la influencia de la suplementación de zinc, puesto que cl número de linfocitos $T$, el estado nutricional y los niveles de fenilalanina sc mantuvieron sin variación durante todo el periodo de suplementación. Los resultados de este estudio sugieren que la respuesta inmune celular podría ser un indicador biológico importante para evaluar la presencia de déficit de zinc en los pacientes fenilquctonúricos.

\section{Resumen}

Existe información contradictoria en relación al estado nutricional de zinc en pacientes con fenilquetonuria. El objetivo de este estudio fue confirmar la existencia de manifestaciones indirectas de déficit de zinc, a través del efecı que ejerce la suplementación de este mineral sobre la respuesta inmune celular. Se determinó la rcacción culánca de hipersensibilidad retardada y la capacidad linfoproliferativa frente a fitohemaglutinina antes y después de suplementación con acetato de zinc, en ocho pacientes fenilquetonúricos. Dos de ocho pacientes presentaron reacción culánea positiva antes de la suplementación, aumentando a seis el número de sujctos con respuesta positiva después de la ingesta de zinc ( $p<0,05$ prucba de Mac Nemar). La capacidad linfoproliferativa aumentó al término de la suplementación con zinc ( $p<0,05$ test de $t$ pareado). Estos resultados sugicren que existe un deficit de nutrición de zinc en pacientes con fenilquetonuria, puesto que la administración de este mineral traza estimularía la respuesta inmune celular.

(Palabras clave: respuesta inmunitaria celular, nutrición zinc, fenilquetonuria.)

\section{Agradecimientos}

Agradecemos a la Srta. Mónica Moraga P. por su cxcelente asistencja técrica y a la Sra. Gloria Jeaza por su valiosa contribución en cl análisis estadístico de los resultados.

\section{Referencias}

1. Gutider F, DiLella $A G$, Ledley FD, et ol.: Molecular biology of phenylkctonuria. Eur J Pediatr 1987; 146 (Strppl 1): A5-A11.

2. Colombo $M$, Troncoso L. Raimann E, Perales CG, Barros T. Cornejo V: Diagnóstico de Fenitguctonura en Chile. Rev Chit Pediatr 1988; 59: 235-239.

3. Williamson $M$; Koch $R$,; Colleen A.; Chong $C$. Correlates of Intelligenoc Test Results in Treated Phenylketonuric Children. Pedialrics 1981: 68: 161-167,

4. Hunt $M$, Berry $H$, White P: Phenylketonuria, adoles. cence, and diet. J Am Dicl Assoc 1985: 85: 1328. 1334.

5. Acosta Ph, Stepnick-Gropper S. Clarke-Sheehan N, et al.: Trace Elcment Status of PKU Children Ingesting an Elemental Diet. JPEN 1987; 11:287-292.

6. Stepnick-Gropper $S$, Acosia $P h$, Clarke-Sheehan $N$, Wenz $E$. Cheng $M$, Koch $R$ : Trace element status of 
children with $\mathrm{PKU}$ and nomal children. J Am Diel Assoc 1988; 88: 459-465.

7. Beissel WR, Edeimat $R$, Nouss $K$, Suskind $R M$ : Single nutrient effects on immunologic functions. JAMA 1981; 245: 53-58.

8. Alien $J, K a y N E, M C C l a i n C J$ : Severe zine deficiency in humans association with a reversible T-lymphocyle dysfunction. Ann In Med 1981; 95: 154157.

9. Weston WI, Huff JC, Humbert JR, Hambidge M: Zinc correction of defective chemolaxis in Acrodematitis Enteropatica Arch Dematol 1977; 113; 422-425.

10. Sandstead HH, Henriksen $L K$, Greger $J L$, Prasad AS, Good RA: Zine nutriture in the elderly in relation 10 taste acuiry, immune response and wound healing. Am J Clin Nutr 1982; 36: 1046-1059.

11. Cunningham-Rundles C, Cunningham-Rundles S, Iwath $T$ et al.: Zing deficiency depressed thymic homones and $T$-lymphocyle dysfunction in patients with hypogammaglobulinemia. Clin Inmunol Immunopathol $1981 ; 21: 387.396$.

12. Guhrie R, Swri A: A Simple Phenylalanine method for detecting phenylketonuria in Large populations of Newborn Infants. Pcdialics 1963; 32: 338-343.

13. Btack R. Lanata CN, Lazo F: Delayed cutaneous bypersensitivity: epidemiologic factors affecting and usefulnessin predicting diartheal incidence in young Peruvian children. Pediatr Infect Dis J 1989; 8: 210215.
14. Oppenheim $J$, Rosentreich DL: Lymphocyte transformation: Utilization of Automatic Cell Harvesters. In: In vitro methods in Cell-Mediated of Tumor Im. munily. Academic Press, New York 1976; 573-585

15. Kung PC, Goldstein G, Reinhertz EL, Schlossmin SF: Monoclonal antibodies defining distinctive human $T$. cell surface antigens. Science 1979; 206: 347-349.

16. Cornejo M, Goya J, Otsen I: Subpoblaciones de linfo. citos T determinadas mediante anticuerpos (AC). Rev Chil Pediatr 1987; 58: 39-43.

17. Passwell J, Gazis E, Efier $T$, Modon $M$, Lilos P, Cohen $E$ : Inmunologic Studies in Phenylketonuria. Acta $P_{c}$ diate Scand 1976; 65: 673-677.

18. Stites $D P$ : Clinical Laboratory Methods of Detecting Cellular İmune Function. En: Fundemberg HH, Stites DP, Caldwell JL, Wells JV (eds). Basic and Clínical Immurology, Los Altos, Califomia: Lange Medicsl Publications, 1980; 382-397.

19. Lonnerdal Bo: Trace Element Nutrition in Infants. Annu Rev Nutr 1989; 9: 109-125

20. Schlesinger L, Arévalo M, Arredondo $S$, Diaz $M$. Lonnerdal B. Slekel A: Effect of a zinc fonified formula on immunocomperence and growth of malnonrished children. Am J Clin Nutr 1992: 56: 491-493.

21. Chropil $\boldsymbol{M}$ : Effect of zinc on cell and biomembranes. Mad Clin North Am 1976; 60: 799-812.

22. Will:ams RO, Loeb LA: Zinc requirement for DNA replication in stinulated buman lymphocytes. J Cebh Biol 1973: 58: 594-601. 